\title{
The Belle II Silicon Vertex Detector
}

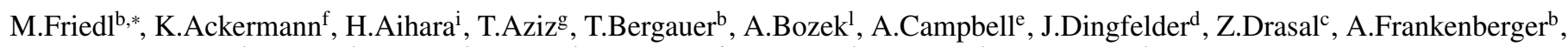

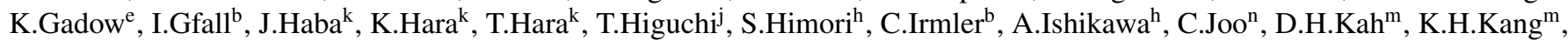

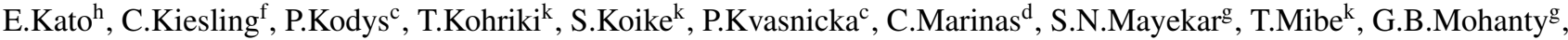

A.Moll $^{\mathrm{f}}$, K.Negishi ${ }^{\mathrm{h}}$, H.Nakayama ${ }^{\mathrm{k}}$, Z.Natkaniec ${ }^{\mathrm{l}}$, C.Niebuhr ${ }^{\mathrm{e}}$, Y.Onuki ${ }^{\mathrm{i}}$, W.Ostrowicz ${ }^{1}$, H.Park $^{\mathrm{m}}$, K.K.Rao ${ }^{\mathrm{g}}$, M.Ritter ${ }^{\mathrm{f}}$, M.Rozanska ${ }^{1}$, T.Saito ${ }^{\mathrm{h}}$, K.Sakai ${ }^{\mathrm{k}}$, N.Sato ${ }^{\mathrm{k}}$, S.Schmid ${ }^{\mathrm{b}}$, M.Schnell ${ }^{\mathrm{d}}$, N.Shimizu ${ }^{\mathrm{i}}$, H.Steininger ${ }^{\mathrm{b}}$, S.Tanakak ${ }^{\mathrm{k}}$, K.Tanida ${ }^{\mathrm{n}}$, G.Taylor ${ }^{\mathrm{a}}$,

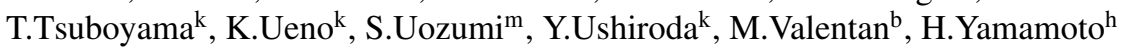

\author{
${ }^{a}$ University of Melbourne, School of Physics, Melbourne, Experimental Particle Physics Group, Parkville Victoria 3010, Australia \\ ${ }^{b}$ HEPHY - Institute of High Energy Physics, Nikolsdorfer Gasse 18, 1050 Vienna, Austria \\ ${ }^{c}$ Charles University, Institute of Particle and Nuclear Physics, Ke Karlovu 3, 12116 Praha 2, Czech Republic \\ ${ }^{d}$ University of Bonn, Department of Physics and Astronomy, Nussallee 12, 53115 Bonn, Germany \\ ${ }^{e}$ DESY, Notkestrasse 85, 22607 Hamburg, Germany

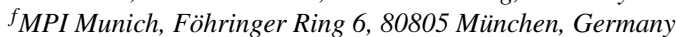 \\ ${ }^{g}$ Tata Institute of Fundamental Research, Experimental High Energy Physics Group, Homi Bhabha Road, Mumbai 400 005, India \\ ${ }^{h}$ Tohoku University, Department of Physics, Aoba Aramaki Aoba-ku, Sendai 980-8578, Japan \\ ${ }^{i}$ University of Tokyo, Department of Physics, 7-3-1 Hongo, Bunkyo-ku, Tokyo 113-0033, Japan \\ ${ }^{j}$ University of Tokyo, Kavli Institute for Physics and Mathematics of the Universe, 5-1-5 Kashiwanoha, Kashiwa, Chiba 277-8583, Japan \\ ${ }^{k}$ KEK, 1-1 Oho, Tsukuba, Ibaraki 305-0801, Japan \\ ${ }^{l}$ Institute of Nuclear Physics, Division of Particle Physics and Astrophysics, ul. Radzikowskiego 152, 31342 Krakow, Poland \\ ${ }^{m}$ Kyungpook National University, Department of Physics, 1370 Sankyuk Dong, Buk Gu, Daegu 702-701, South Korea \\ ${ }^{n}$ Seoul National University, High Energy Physics Laboratory, 25-107 Shinlim-dong, Kwanak-gu, Seoul 151-742, South Korea
}

\begin{abstract}
The KEKB machine and the Belle experiment in Tsukuba (Japan) are now undergoing an upgrade, leading to an ultimate luminosity of $8 \times 10^{35} \mathrm{~cm}^{-2} \mathrm{~s}^{-1}$ in order to measure rare decays in the B system with high statistics.

The previous vertex detector cannot cope with this 40 -fold increase of luminosity and thus needs to be replaced. Belle II will be equipped with a two-layer Pixel Detector surrounding the beam pipe, and four layers of double-sided silicon strip sensors at higher radii than the old detector. The Silicon Vertex Detector (SVD) will have a total sensitive area of $1.13 \mathrm{~m}^{2}$ and 223,744 channels twice as many as its predecessor.

All silicon sensors will be made from $150 \mathrm{~mm}$ wafers in order to maximize their size and thus to reduce the relative contribution of the support structure. The forward part has slanted sensors of trapezoidal shape to improve the measurement precision and to minimize the amount of material as seen by particles from the vertex. Fast-shaping front-end amplifiers will be used in conjunction with an online hit time reconstruction algorithm in order to reduce the occupancy to the level of a few percent at most. A novel "Origami" chip-on-sensor scheme is used to minimize both the distance between strips and amplifier (thus reducing the electronic noise) as well as the overall material budget.

This report gives an overview on the status of the Belle II SVD and its components, including sensors, front-end detector ladders, mechanics, cooling and the readout electronics.
\end{abstract}

Keywords: Silicon, Vertex Detector, Belle II, Origami

\section{Introduction}

The Belle detector [1] was the only experiment at the highluminosity (peak value was $2.11 \times 10^{34} \mathrm{~cm}^{-2} \mathrm{~s}^{-1}$ ) KEKB asymmetric electron-positron collider, operating from 1999 to 2010 at KEK in Tsukuba (Japan). Its large data sample confirmed the $\mathrm{CP}$ violation in the $\mathrm{B}$ meson system and was therefore also mentioned by the Nobel committee at the 2008 award to M. Kobayashi and T. Maskawa.

Even more luminosity is needed to study rare decays with high precision and to hunt for faint signatures of new physics.

*Corresponding author, Email: markus.friedl@oeaw.ac.at
Thus, an upgrade program was launched to build a machine and an experiment with a design luminosity that is 40 times higher than previously achieved. This obviously requires significant refurbishment of all parts and in particular of the vertex detector, which will be replaced by an entirely new device.

As in Belle, the Silicon Vertex Detector (SVD) of the Belle II experiment [2] will be composed of four layers of double-sided silicon detectors with orthogonal strips on opposing sides. It will be shifted toward higher radii and complemented by a new two-layer Pixel Detector (PXD), which is described in another article in this volume [3].

Since KEKB and its successor SuperKEKB are low-energy machines operating mostly at the $\Upsilon(4 S)$ resonance, minimiza- 
tion of the material budget in the active volume is of utmost importance to limit multiple scattering and thus to achieve meaningful physics results. Consequently, the largest possible sensor size - made from $150 \mathrm{~mm}$ silicon wafers - was chosen to reduce the relative amount of material in the support structure, which is composed of a light-weight carbon fiber/foam core sandwich.

The readout electronics must be much faster than before in order to cope with the luminosity increase and to maintain manageable levels of occupancy and pile-up. Moreover, a pipelined readout is required to avoid dead-time. Unfortunately, shorter integration time of the front-end amplifier also implies a higher noise figure, which mainly depends on the input capacitance. Thus, it is necessary to place the front-end chips as close as possible to the sensor strips, i.e. in the active region. We have developed the so-called "Origami" chip-on-sensor ladder design [4] which provides a light-weight solution to these conflicting requirements. This includes thin pipes with two-phase $\mathrm{CO}_{2}$ cooling operating at $-20^{\circ} \mathrm{C}$.

Using a special mode of the APV25 front-end chip [5], which was originally developed for the for the tracker of the CMS experiment at the LHC, we are able to reconstruct the timing of a particle with a precision of a few ns, allowing to discard the off-time background which is inevitably present in the system. This adds an excellent timing accuracy to the good spatial resolution of the SVD, which is however prone to ambiguities due to ghost hits (combinatorics of multiple hit strips on the two sensor sides leading to true and false intersection points). On the other hand, the PXD will provide unambiguous hit coordinates, but has a large integration time and thus delivers a significant amount of background data. The full power of the Belle II Vertex Detector $(\mathrm{VXD}=\mathrm{PXD}+\mathrm{SVD})$ results from the combination of both data streams. Online SVD tracking will be performed for the benefit of PXD data reduction.

\section{Front-End}

\subsection{Geometry}

Figure 1 shows a rendering of the Belle II SVD with only one ladder equipped per layer. Moreover, the shaded cones mark the limits of the sensitive volume which covers a polar angle range of $17^{\circ}$ to $150^{\circ}$ and is consistent with other subdetectors. Thanks to this limited acceptance, heavy and bulky structures can be placed outside, where in fact also heavy metal shields will be placed to reduce the amount of unwanted background radiation.

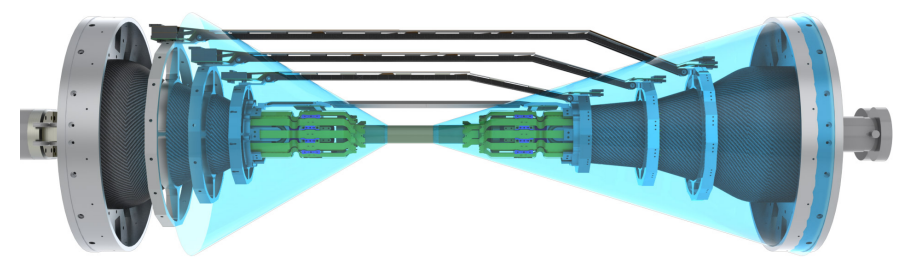

Figure 1: Rendering of the SVD with transparent cones indicating the borders of the active volume. Just a single ladder is shown in each layer.

The acceptance region is larger on the right side due to the asymmetric energies of colliding beams, creating a forward boost that is essential for precise measurement of secondary vertices. It also influenced the SVD ladder design, containing a slanted sensor in the forward part for all layers except the innermost one. This results in a reduced overall number of silicon sensors and readout channels as well as less material (and thus multiple scattering) in the trajectories of particles in that region. This, however, comes at the cost of a more complicated structure and assembly procedure and also has implications for tracking.

Table 1 summarizes the design specifications of the SVD for the four layers. The numbering starts with three in order to exclude potential confusion with the two PXD layers, resulting in an unambiguous VXD numbering scheme.

\begin{tabular}{|l|c|c|c|}
\hline Layer & $\begin{array}{c}\text { Radius } \\
{[\mathrm{mm}]}\end{array}$ & Ladders & $\begin{array}{c}\text { Sensors } \\
\text { per Ladder }\end{array}$ \\
\hline L3 & 38 & 7 & 2 \\
\hline L4 & 80 & 10 & 3 \\
\hline L5 & 104 & 12 & 4 \\
\hline L6 & 135 & 16 & 5 \\
\hline
\end{tabular}

Table 1: Layer configuration of the Belle II SVD.

\subsection{Silicon Sensors}

The SVD geometry was optimized to be equipped with a limited number of sensor variants. As a result, there are only three different sensor designs, as summarized in Table 2. The thickness of all sensors is about $300 \mu \mathrm{m}$. The p-side strip implants run along the length of the sensor in parallel to and facing the beam axis. Consequently, n-side strips are shorter and approximately tangential to the barrel circumference at the respective radius.

\begin{tabular}{|l|c|c|c|c|}
\hline Name & $\begin{array}{c}\text { Length } \\
{[\mathrm{mm}]}\end{array}$ & $\begin{array}{c}\text { Width } \\
{[\mathrm{mm}]}\end{array}$ & $\begin{array}{c}\text { p-pitch } \\
{[\mu \mathrm{m}]}\end{array}$ & $\begin{array}{c}\text { n-pitch } \\
{[\mu \mathrm{m}]}\end{array}$ \\
\hline Small rectangular & 122.8 & 38.4 & 50 & 160 \\
\hline Large rectangular & 122.8 & 57.6 & 75 & 240 \\
\hline Trapezoidal & 122.8 & $57.6-38.4$ & $75-50$ & 240 \\
\hline
\end{tabular}

Table 2: Properties of the three sensor designs used in the Belle II SVD. Length and width values refer to the active sensor area, where about $1 \mathrm{~mm}$ of inactive material is to be added on each edge.

The small rectangular sensor is only used in the innermost layer (L3) and therefore has a smaller pitch to increase the hit resolution toward the PXD. All other layers (L4-L6) use ladders composed of a variable number of large rectangular sensors in the barrel region and one trapezoidal sensor in the slanted forward part. Ladders are tilted with respect to the tangential plane by $5-7^{\circ}$ (depending on the layer) and overlap their neighbors in a windmill-like structure in order to improve the alignment and compensate for Lorentz shifts in the magnetic field of $1.5 \mathrm{~T}$.

In total, the SVD is composed of 172 double-sided silicon sensors with a sensitive area of $1.13 \mathrm{~m}^{2}$ and 223,744 strips which are read out by 1,748 APV25 front-end chips. 
The sensor design was verified in beam tests and samples were irradiated with gammas. A detailed study of n-strip isolation using p-stop and p-spray techniques with various geometries was performed [6]. Subsequently, the best performing variant according to the conditions in Belle II was chosen for the series production.

All rectangular sensors are manufactured by Hamamatsu Photonics K.K. (Japan), while the trapezoidal sensors are produced by Micron Semiconductor Ltd. (UK). The majority of installed sensors is of large rectangular type, and their production is already completed including some spares. The other two types were already ordered, are now in production and will be fully delivered by the end of 2013 .

\subsection{Origami Chip-on-Sensor Concept}

As mentioned before, the front-end amplifiers must be close to the readout strips of the silicon sensors. This can be achieved in a conventional way for those sensors that are located at the edge, using hybrid boards (which are in fact standard printed circuit boards) and pitch adapters. This approach, however, is not suitable for the inner detectors of a ladder. There, we apply the Origami chip-on-sensor concept, where the hybrid board is replaced by a thin flex which sits on top of the sensor. The top side strips are attached to short fanouts, while the bottom side strips are connected to pitch adapters which are bent around the edge of the sensor - hence the name Origami.

Details about the Origami concept, module assembly and beam test results can be found in Reference [4].

\section{Electronics}

\subsection{Overview}

A simplified overview of the SVD readout chain [7] is shown in Fig. 2. The front-end contains readout hybrids with four or six APV25 chips arranged either on Origami flex circuits or conventional PCBs for the edge sensors. These are connected by miniature twisted flat-band cables to Junction Boxes which are located about $2 \mathrm{~m}$ away from the front-end. Those boxes serve as patch panels and bring in the power to the front-end by using rad-hard DC/DC converters designed by CERN [8]. Subsequently, the signals are transmitted to the FADC modules using the same twisted flat-band cable as before in order to avoid excessive reflections.

The FADC modules are hosted in 9U VME crates and are steered by a controller module. A Finesse Transmitter Board (FTB) is attached on the rear side of each FADC module, which sends out the data over two parallel optical links. One of these leads to the COPPER module, which is already part of the common Data Acquisition (DAQ) for Belle II. A copy of the same data stream is sent to a Data Concentrator (DATCON), which reconstructs tracks from SVD segments online. These tracks are then extrapolated to the PXD to create Regions of Interest (ROI) around the intersection points with the pixel sensor planes. Thus, the amount of PXD data can immediately be reduced by discarding background hits which do not correspond to any SVD tracks.

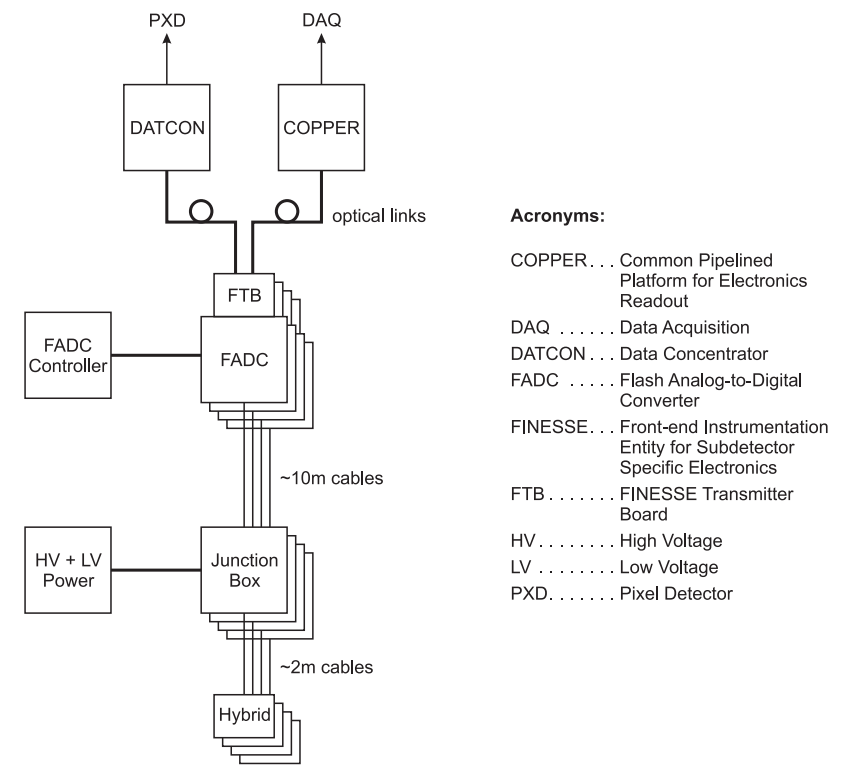

Figure 2: Simplified view of the Belle II SVD readout chain. The data flow direction is from bottom to top.

\subsection{Junction Box}

Junction Boxes (Fig. 3) will be installed on both forward and backward sides of Belle II outside of the acceptance volume. These have a size like a shoe box and contain six plug-in printed circuit boards. The copper bottom plate will serve as a heat sink that is cooled with water. As the Junction Box will be in a radiation zone, it does not contain any active electronics except for the aforementioned DC/DC converter modules which can tolerate much higher radiation levels than expected for this application. Moreover, they use a shielded toroidal air coil and thus can operate in the magnetic field. They are thermally connected to the heat sink.

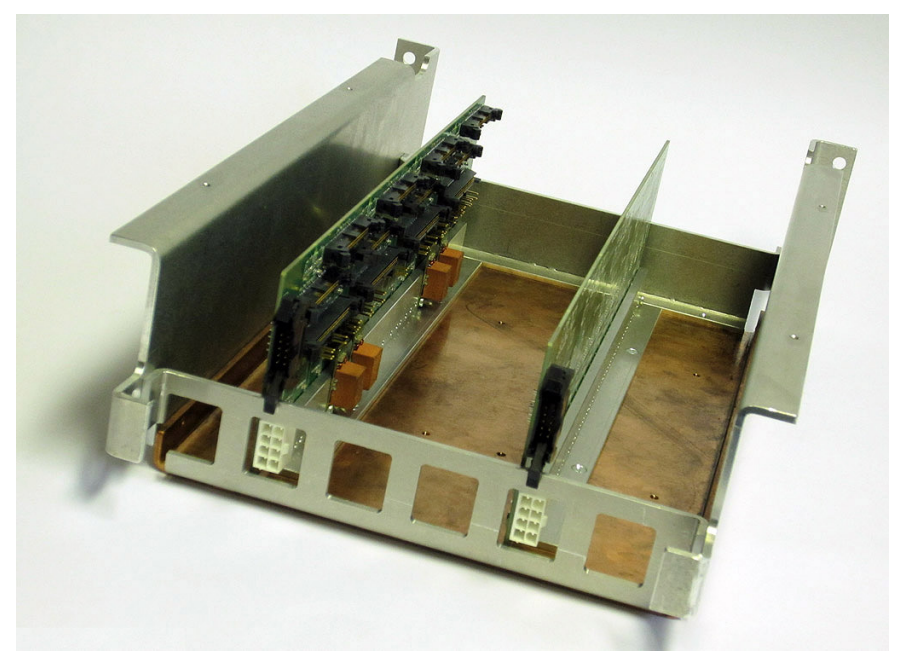

Figure 3: Prototype Junction Box with one plug-in board and one dummy.

\section{3. $F A D C$}

The Belle II SVD back-end electronics will consist of four 9U VME crates located on top of the experiment, which will 
contain 48 FADC modules. Those receive the analog front-end signals, perform a DC level translation (the front-end APV25 electronics LV power is tied to the \pm bias voltage levels) and digitize the signals. Subsequently, data processing is done individually for each input channel inside a powerful Altera Strativ IV GX FPGA, starting with an FIR filter for signal conditioning, followed by frame detection and extraction, data reordering, pedestal subtraction, a two-pass common mode correction, zero suppression and eventually hit-time finding. More details on the FADC functionality can be found in [7].

For the sake of modularity, some parts of the FADC circuitry are designed as plug-in boards. Analog and digital level translation circuits are implemented as double-sided daughter boards to increase the density of readout channels per module. The central FPGA is also hosted on a daughter board which is shown in Fig. 4
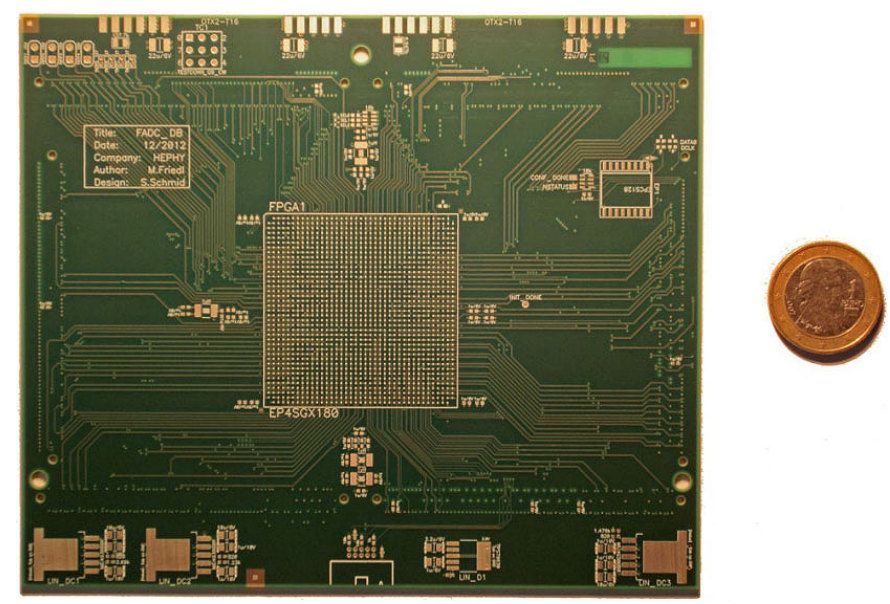

Figure 4: Daughter board hosting the central Altera Stratix IV GX FPGA.

\subsection{FTB}

The FTB is a Eurocard sized board which is plugged into the P3 connector on the rear of each FADC module. It converts the processed FADC output data to two identical optical links, which will lead to the common DAQ and the PXD detector, respectively. In 2012, the Belle2Link firmware for the optical links was successfully transferred to the FTB FPGA and operated at various speeds on FTB prototype boards.

\section{Performance}

Thanks to the ability of reading out several samples along the shaped front-end amplifier waveform, we can reconstruct the actual particle timing with high precision by applying a fit function to each hit n-tuple. This fit function is actually a reference curve recorded in the calibration mode of the APV25 chip and has two parameters: amplitude and time shift. In the future, this method will be integrated in the FPGA of the FADC using lookup tables, which were already confirmed to yield a similar precision as the numerical fit.

In the past years, beam test measurements were performed on various prototype detector modules using several detector geometries, covering a broad range of signal-to-noise ratios. When comparing the fitted hit time to a reference TDC, it turns out that the accuracy of the timing inversely scales with the signal-to-noise ratio [9], as shown in Fig. 5]

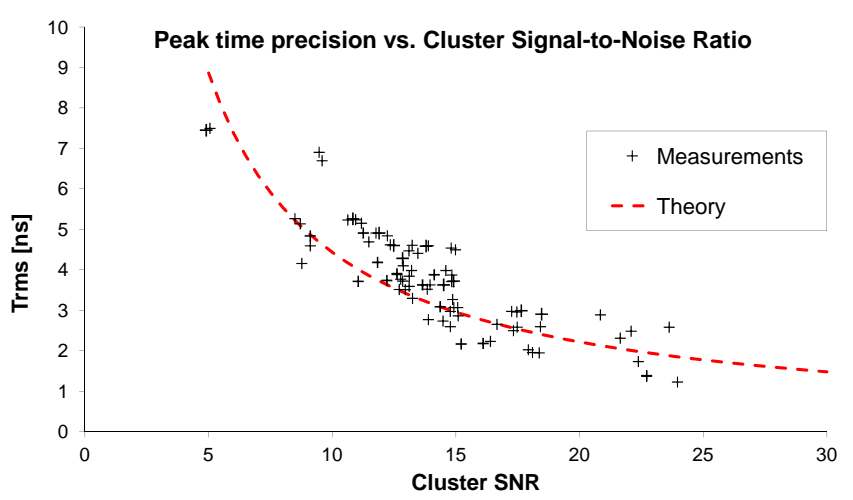

Figure 5: RMS hit time finding accuracy vs. cluster signal-to-noise. The theoretical prediction is taken from [9].

\section{Summary and Outlook}

The four-layer geometry of the Belle II Silicon Vertex Detector has been worked out in detail, using three different doublesided sensor designs. The majority of those were delivered already, others will follow by the end of 2013. A prototype of the final back-end readout electronics is now being built and used for firmware development. It has already been shown that a time resolution of a few nanoseconds can be achieved, which will improve tracking and allows to discard off-time background hits. The SVD data will be sent to the Pixel Detector, where they will be used for online data reduction thanks to their excellent timing.

Presently, the SVD undergoes a transition from R\&D phase to final prototyping and mass production. According to the current schedule, the SVD - both front-end and back-end parts will be completed in 2015 and installed in Belle II.

\section{References}

[1] A. Abashian et al. (The Belle Collaboration), The Belle Detector, Nucl. Instr. and Meth. A 479 (2002) 117-232

[2] Z. Doležal, S. Uno (editors), Belle II Technical Design Report, KEK Report 2010-1, arXiv:1011.0352

[3] J. Schieck, DEPFET pixels as a vertex detector for the Belle II experiment, this volume

[4] C. Irmler et al. A Low Mass On-chip Readout Scheme for Double-Sided Silicon Strip Detectors, this volume

[5] M. French et al., Design and results from the APV25, a deep sub-micron CMOS front-end chip for the CMS tracker, Nucl. Instr. and Meth. A 466 (2001) 359-365

[6] M. Valentan et al., Performance Studies on the Ohmic Side of Silicon Microstrip Sensors, this volume

[7] M. Friedl et al., The Belle II Silicon Vertex Detector readout chain, JINST 7 C02037 (2012)

[8] S. Michelis et al., DC-DC converters in $0.35 \mu \mathrm{m}$ CMOS technology, JINST 7 C01072 (2012)

[9] G. de Geronimo, Low-Noise Electronics for Radiation Sensors, in: K.Iniewski (editor), Medical Imaging: Principles, Detectors, and Electronics, John Wiley \& Sons (2009) 